\title{
Urinary Tract Infection In Sobrata, Algmel Cities In Libya 2013
}

\author{
Khalifa AA Fatnasa ${ }^{1^{*}}$, Alaa Othman Harb ${ }^{1}$ and Abdalhamed M Alkout ${ }^{2}$ \\ ${ }^{1}$ National Board for Vocational and Technical Education in Tripoli, Libya \\ ${ }^{2}$ Faculty of Medical Technology, University of Tripoli, Libya
}

*Corresponding author: Khalifa AA Fatnasa, Faculty of Medical Technology, High Institute of Comprehensive Vocations in Tiji City, National Board for Vocational and Technical Education in Tripoli, Libya, Tel: 00218918722625; E-mail: kbf_2008@yahoo.com

Rec date: Jun 12, 2014; Acc date: Jul 22, 2014; Pub date: Jul 30, 2014

Copyright: (c) 2014 Khalifa AAF, et al. This is an open-access article distributed under the terms of the Creative Commons Attribution License, which permits unrestricted use, distribution, and reproduction in any medium, provided the original author and source are credited.

\begin{abstract}
:
Urinary tract infections are very common particularly in women, babies and the elderly. About one in two women, and one in $\mathbf{2 0}$ men will get the infection in their lifetime. The majority of urinary tract infections are urethritis, cystitis, and pyeleonephritis. The most common bacteria causes the infection is a bacterium common to the digestive tract called Escherichia coli. The most common symptoms of the infection are, burning sensation during urination, blood in urine, and Lower abdominal pain, therefore the infection needs to be investigated as it may indicates more serious conditions. A study with patients, who have come with the symptoms of urinary tract infection in the cities of Sobrata, and Algmel in Libya has been made the number of patients in the study were 582 cases and 926 cases respectively. After the investigations have been made the results shows those 301 women, 66 men in Sobrata city and 354 women, 171 men in Algmel city were infected. The percentage of infection are $63 \%$ and $56.69 \%$. Number of women and men who infected by Escherichia coli are $(165,46)$ and $(179,90)$ cases respectively, this result approves that bacteria Escherichia coli is the major causes of urinary tract infection symptoms in most of the patients. The appropriate antibiotics for the infection are Augmentines and Ciprofloxacin based on the study.
\end{abstract}

Keywords: Urinary Tract Infections (UTI); Escherichia coli; Urethritis; Cystitis; Pyeleonephritis

\section{Introduction}

Urinary tract infections are a serious health problem affecting millions of people each year. Infections of the urinary tract are the second most common type of infection in the body [1]. It is the most common urological disease in the United States with a total annual cost of more than $\$ 3.5$ billion [2]. It is one of the most common bacterial infections affecting humans being throughout their life span, it is account for more than 8 million visits to physician's offices, 1.5 million emergency room visits, and 300,000 hospital admissions in the United States annually [3,4]. The annual incidence of UTI in French is 53,000 diagnoses per million persons per year, which represents $1.05 \%$ to $2.10 \%$ of the activity of general practitioners [5]. In the United States the annual number of diagnoses of pyelonephritis in females was estimated to be 250,000 [5]. These infections are more common in females than in male, and the incidence in women in the age of 20- 40 years ranges from 25 to $30 \%$ whereas in elderly patients, older women above 60 years of age, it ranges from 4 to $43 \%$ [6,7]. Urinary tract infections are the frequent infections observed in clinical practice and results in a significant morbidity and high medical costs, it is a common infection observed in diabetic patients, which alters the genitourinary system where UTI can be a cause of severe complications ranging from dysuria (pain or burning sensation during Urination) organ damage and sometimes even death due to (pyeleonephritis) [8]. The risk factors for UTI involve colonization with a different uropathogen in cases of recurrent UTI, glucosuria and impaired granulocyte function [9]. Urinary tract infection is the second most common clinical indication for empirical antimicrobial treatment in primary and secondary care [10]. Urine samples constitute the largest single category of specimens examined in most medical microbiology laboratories [10,11]. The diagnosis of UTI is particularly difficult in elderly patients, who are more likely to have asymptomatic bacteriuria as they get older. UTIs can be classified as uncomplicated or complicated [11-13]. Elderly institutionalized patients frequently receive unnecessary antibiotic treatment for asymptomatic bacteriuria despite clear evidence of adverse effects with no compensating clinical benefit $[14,15]$. Approximately 1 in 3 women will require antimicrobial treatment for a urinary tract infection before age 24 , and $40 \%$ to $50 \%$ of women will have a UTI during their life time. Escherichia coli is found to be the most common cause of UTIs and treatment should be based on urine culture [16]. Infections of the urinary tract are among the most common infections in the hospital if not treated promptly and appropriately, and may lead to significant acute morbidity and irreversible renal damage. Children, however, have a wide variety of clinical presentation, ranging from the asymptomatic presence of bacteria in the urine to potentially life threatening infection of the kidney. The main goals are early diagnosis, and appropriate antimicrobial therapy. The purpose was to evaluate the prevalence of UTI among Sobrta city patients and compare them with those from Algmel city patients.

\section{Material and Methods}

Clean midstream urine samples were collected in sterile tubes from 582 patients in Sobrata hospital in Sobrata city and from 926 patients in Dar Alshruk clinic in Algmel city in 2013, those patients have come with the symptoms of urinary tract infection, urine samples were transported in ice and processed in the laboratory within $2 \mathrm{hrs}$ of collection by using urine analysis and routine microbiological techniques. The patients were educated about the collection of urine sample to avoid contamination. All urine samples were plated with 100 $\mu \mathrm{l}$ of urine sample using standard pour plate techniques on nutrient agar, blood agar, MacConkey agar (Difco, Kansasa, USA), and 
Page 2 of 3

incubated at $37^{\circ} \mathrm{C}$ overnight for visible growth and to isolate the microbial agents of UTI. Urine samples showing colony count more than $10,000 \mathrm{Cfu} / \mathrm{ml}$ was considered to be significant for urinary tract infections [17]. UTIs isolates were identified following standard biochemical tests. Antimicrobial susceptibility testing was done on Mueller-Hinton agar using disk diffusion technique. This method was done according to Clinical and Laboratory Standards Institute (CLSI) guidelines (2002) to determine susceptibility of UTIs agents [18]. The antibiotics discs used are Ciprofloxacin, Augmentines, Amoxicillin, Penicillin, Kanamycine. The UTIs isolates showed sensitivity against Ciprofloxacin, Augmentines, Amoxicillin in both cities which means they are the proper antibiotics should be prescribed to the patients by physician. Identification of $S$. saprophyticus is made on the basis of the absence of hemolysin and coagulase and resistance to novobiocin. Novobiocin susceptibility test results are $100 \%$ sensitive and $96 \%$ specific. $S$. saprophyticus is innately resistant to the antibiotic novobiocin. Therefore, screening coagulase-negative staphylococci from urine cultures for novobiocin resistance is a reliable presumptive identification of Staphylococcus saprophyticus [19].

\section{Results}

A study to the patients, who have come with the symptoms of urinary tract infection in the cities of Sobrata and Algmel, in 2013, has been made, the number of patients in the study were 582 and 926 cases respectively. After the investigations made by using routine microbiological techniques, the results shows that the percentage of infection in two cities were $63 \%$ and $56.69 \%$ respectively, and those 301 female (51.72\%), 66 male (11.34\%) in Sobrata city, and 354 female (38.2\%), 171 male (18.5\%) in Algmel city were infected from total cases. No urinary tract infection was found in 111 female (19.07\%), 104 male (17.87\%) in Sobrata city, and 270 female (29.2\%),131 male (14.1\%) in Algmel city.UTI isolates in males and females in both cities included Escherichia coli 165 (28.35\%) female,46 (7.90\%) male in Sobrata city and179 (19.33\%) female, 90 (9.7\%) male in Algmel city, following Staphylococcus saprophyticus $83(14.26 \%)$ female, $11(1.9 \%)$ male, in Sobrata city and $74(7.99 \%)$ female, $38(4.1 \%)$ male in Algmel city, following Klebsiella pneumoniae 37(6.36\%) female, 6(1.03\%) male, in Sobrata city and 66(7.12\%) female, 29(3.13\%) male inAlgmel city, and followed by Streptococcus faecalis $16(2.75 \%)$ female, 3(0.52\%) male in Sobrata city and 35 (3.77\%) female, 14(1.51\%) male in Algmel city, this result approves that bacteria Escherichia coli was the most common prevalent causes of UTI in most of the cases in Sobrata and Algmel cities, also the infection is more frequent in female than male and more in Sobrata city than in Algmel city in Libya (Table 1-3).

\begin{tabular}{|l|l|l|l|l|}
\hline UTI pathogen & $\begin{array}{l}\text { Prevalence of UTI (Female) } \\
(\%)\end{array}$ & Prevalence of UTI (Male) (\%) & Total infection (\%) & Non-infected (\%) \\
\hline Percent of infections & $301(51.72 \%)$ & $66(11.34 \%)$ & $367(63.06 \%)$ & $215(36.94 \%)$ \\
\hline Escherichia coli & $165(28.35 \%)$ & $46(7.90 \%)$ & $211(36.25 \%)$ & \\
\hline Staphylococcus saprophyticus & $83(14.26 \%)$ & $11(1.89 \%)$ & $94(16.15 \%)$ & \\
\hline Klebsiella pneumoniae & $37(6.36 \%)$ & $6(1.03 \%)$ & $43(7.39 \%)$ & \\
\hline Streptococcus feacalis & $16(2.75 \%)$ & $3(0.52 \%)$ & $19(3.27 \%)$ & \\
\hline
\end{tabular}

Table 1: Bacterial species in UTI according to the sex in 367 patients from Sobrat city -shows the prevalence of UTI pathogens according to sex males and females. Commonly recovered UTI pathogens in female and male included E. coli 165 (28.35\%), 46 (7.90\%), Staphylococcus saprophyticus 83 (14.26\%), 11 (1.89\%), Klebsiella pneumoniae 37 (6.36\%), 6 (1.03\%), Staphylococcus feacalis 16 (2.75\%) $3(0.52 \%)$.

\begin{tabular}{|l|l|l|l|l|}
\hline UTI pathogen & Prevalence of UTI (Female) (\%) & Prevalence of UTI (Male) (\%) & Total infection (\%) & non infected (\%) \\
\hline Percent of infections & $354(38.2 \%)$ & $171(18.3 \%)$ & $525(56.7 \%)$ & $401(43.3 \%)$ \\
\hline Escherichia coli & $79(19.33 \%)$ & $90(9.72 \%)$ & $169(29.05 \%)$ & \\
\hline Staphylococcus saprophyticus & $74(7.99 \%)$ & $38(4.10 \%)$ & $112(12.09 \%)$ & \\
\hline Klebsiella pneumoniae & $66(7.12 \%)$ & $29(3.13 \%)$ & $95(10.25)$ & \\
\hline Streptococcus feacalis & $35(3.77 \%)$ & $14(1.51 \%)$ & $49(5.28)$ & \\
\hline
\end{tabular}

Table 2: Bacterial species in UTI according to the sex in 525 patients from Algmel city -shows the prevalence of UTI pathogens according to sex males and females. Commonly recovered UTI pathogens in female and male included E.coli 179 (19.33\%) 90 (9.72\%), Staphylococcus saprophyticus 74 (7.99\%) 38 (4.10\%, Klebsiella pneumoniae 66 (7.12\%)29 (3.13\%), Staphylococcus feacalis 35 (3.77\%) 14 (1.51\%). 


\begin{tabular}{|l|l|}
\hline Eschericia coli & Ciprofloxacin, Augmentines, Amoxicillin \\
\hline Staphylococcus saprophyticus & Ciprofloxacin, Augmentines \\
\hline Klebsiella pneumonia & Ciprofloxacin,Augmentines \\
\hline Streptococcus faecalis & Ciprofloxacin, Augmentine \\
\hline
\end{tabular}

Table 3: The appropriate antibiotics for UTIs isolates in the study based on Antimicrobial susceptibility testing

By looking at Table 3, we could realize that the most effective antibiotic for the most of the UTIs isolates were Ciprofloxacin, and Augmentine.

\section{Discussion and Conclusion}

In this study 582 and 926 samples with a presumptive diagnosis of bacterial urinary tract infection were analyzed. The prevalence of acute bacterial UTI was $63 \%$ in Sobrata city and $56.69 \%$ in Algmel city respectively, female were more affected by the infection than male, except with klebsiella pneumoniae, and Streptococcus faecalis male were more affected, and the infections were more predominant in Sobrta city than Algmel city. The total infection of male to female ratio was 1:4 in Sobrat city and 1:2 in Algmel city. The prevalence of Escherichia coli in Sobrta and Algmel respectivelyis (36.25\%) (29.05\%) which make it the predominant organism identified, followed by Staphylococcus saprophyticus (16.15\%) (12.09\%), followed by klebsiella pneumoniae (7.39\%) (10.25\%), followed by Streptococcus faecalis $(3.27 \%)(5.28 \%)$, and a little Candida Spp, this results compatible with the fact that $E$. coli is the most common causes of urinary tract infection. We come out with the study to diffuse awareness about the causes of UTI, and to raise awareness of the risk of giving antibiotics and their direct impact in the outcome analysis of urine tract infections.

\section{Acknowledgments}

The authors thank all technician in Alrazy and Dar Alshoruk clinic laboratory for their assistance in collecting and handling samples and to all students who have helped in the work; Amal Hoyge, Safa omar, Maroua Hoyge, Najat Mrabat, Heba sallam, Hager Shoia,Raja Shoia, Hamida Alzahani, Eman Ambarak.

\section{References}

1. Morgan MG, McKenzie H (1993) Controversies in the laboratorydiagnosis of community-acquired urinary tract infection. Eur JClinMicrobiol Infect Dis12: 491-504.

2. Gaymans R, Haverkorn MJ, Valkenburg HA, Goslings WR (1976) Aprospective study of urinary-tract infections in a Dutch general practice. Lancet 2: 674-677.

3. Nicolle LE (2000) Asymptomatic bacteriuria in institutionalized elderly people: evidence and practice. CMAJ 163: 285-286.

4. Walker S, McGeer A, Simor AE, Armstrong-Evans M, Loeb M (2000) Why are antibiotics prescribed for asymptomatic bacteriuriain institutionalized elderly peopleA qualitative study ofphysicians' and nurses' perceptions. CMAJ 163: 273-277.

5. Geerlings SE (2008) Urinary tract infections in patients with diabetes mellitus: epidemiology, pathogenesis and treatment. Int J Antimicrob Agents 31 Suppl 1: S54-57.

6. Stamm WE, Hooton TM (1993) Management of urinary tract infections in adults. N Engl J Med 329: 1328-1334.

7. Pinson AG1, Philbrick JT, Lindbeck GH, Schorling JB (1994) ED management of acute pyelonephritis in women: a cohort study. Am J Emerg Med 12: 271-278.

8. Litwin MS, Saigal CS, Yano EM, Avila C, Geschwind SA, et al. (2005) Urologic diseases in America Project: analytical methods and principal findings. J Urol 173: 933-937.

9. Jarvis WR, Martone WJ (1992) Predominant pathogens in hospital infections. J Antimicrob Chemother 29 Suppl A: 19-24.

10. Williams DH1, Schaeffer AJ (2004) Current concepts in urinary tract infections. Minerva Urol Nefrol 56: 15-31.

11. Mittal R, Chhibber S, Sharma S, Harjai K (2004) Macrophage inflammatory protein-2, neutrophil recruitment and bacterial persistence in an experimental mouse model of urinary tract infection. Microbes Infect 6: 1326-1332.

12. Nicolle LE (2008) Uncomplicated urinary tract infection in adults including uncomplicated pyelonephritis. Urol Clin North Am 35: 1-12, v.

13. Mittal R, Aggarwal S, Sharma S, Chhibber S, Harjai K (2009) Urinary tract infections caused by Pseudomonas aeruginosa: a minireview. J Infect Public Health 2: 101-111.

14. Foxman B (2003) Epidemiology of urinary tract infections: incidence, morbidity, and economic costs. Dis Mon 49: 53-70.

15. MehvishSaleem, Betty Daniel (2011) Prevalence of Urinary Tract Infection among Patients with Diabetes in Bangalore City. Int. J. Emerg.Sci 1: 133-142.

16. Clinical and laboratory standards institute. Performance standards for antimicrobial susceptibility testing.12th Informational supplement. CLSI document M100-S12.Pennsylvania, USA. 2002; 1 : 1.

17. Mansour Amin, ManijehMehdinejad, ZohrehPourdangchi (2009) Study of bacteria isolated from urinary tract infections and determination of their susceptibility to antibiotics. Jundishapur Journal of Microbiology 2: 118-123.

18. Wilson ML, Gaido L (2004) Laboratory diagnosis of urinary tract infections in adult patients. Clin Infect Dis 38: 1150-1158.

19. Adriano Martison Ferreira, Mariana Fávero Bonesso, Alessandro Lia Mondelli, Maria de Lourdes Ribeiro de Souza da Cunha (2012) Identification of Staphylococcus saprophyticus isolated from patients with urinary tract infection using a simple set of biochemical tests correlating with $16 \mathrm{~S}-23 \mathrm{~S}$ interspace region molecular weight patterns. J of Microbiol Methods 91: 406-411. 